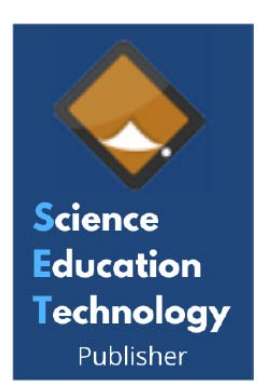

\title{
Cefdinir Inclusion in Mesoporous Silica as Effective Dissolution Enhancer with Improved Physical Stability
}

\author{
Raghad Al Nuss* and Hind El Zein \\ Department of Pharmaceutics and Pharmaceutical Technology, Faculty of Pharmacy, Damascus University, \\ Damascus, Syria
}

\author{
Article Info: \\ Keywords: \\ Cefdinir, \\ dissolution rate enhancement, \\ mesoporous silica, \\ SBA-15, \\ drug release kinetics, \\ improved physical stability. \\ Timeline: \\ Received: August 25, 2021 \\ Accepted: October 05, 2021 \\ Published: October 21, 2021 \\ Citation: Al Nuss R, El Zein H. Cefdinir \\ Inclusion in Mesoporous Silica as \\ Effective Dissolution Enhancer with \\ Improved Physical Stability. J Pharm \\ Nutr Sci 2021; 11: 73-86.
}

\begin{abstract}
:
Objective: The objective of this research was to enhance the physical stability and the dissolution rate of cefdinir, a BCS class IV drug, characterized by low and variable bioavailability due to both its low solubility and low permeability.

Methods: Cefdinir was loaded into the mesoporous silica (SBA-15), by using the solvent immersion method starting from different organic solvents. And then formula (F3), which exhibited the highest loading percentage, was selected to study its drug release in media with different $\mathrm{pH}(1.2,4.5$, and 6.8), and has been fully characterized by using: Fourier Transform Infrared Spectroscopy (FT-IR) Spectroscopy, Differential Scanning Calorimetry, Powder X-ray Diffraction, and has been subjected to accelerated stability tests using different temperatures and relative humidity. Drug release kinetics were studied by using the following models: Probit, Gompertz, Weibull, and Logistic.

Results: The results showed a remarkable dissolution improvement of cefdinir from the loaded silica in comparison to the crystalline drug at the different studied media. Drug release behaviors were well simulated by the Weibull model for F3 in all studied media and for pure Cefdinir in phosphate buffer only, and by the Gompertz function for pure Cefdinir in $\mathrm{HCl}$ buffer and Acetate buffer. FTIR results showed hydrogen bonds formed between the drug and silica, DSC and PXRD results revealed the transformation of cefdinir into an amorphous form upon adsorption. Stability studies under different conditions revealed the ability of mesoporous silica to maintain the amorphous state of the drug, which has been formed upon adsorption, and to prevent re-organization in the crystal nucleus of the drug molecules.
\end{abstract}

Conclusion: Thus, loading cefdinir onto mesoporous silica can be used as a promising method to enhance drug dissolution, and maintain the physical stability of its amorphous form.

DOI: https://doi.org/10.29169/1927-5951.2021.11.10

${ }^{*}$ Corresponding Author

Tel: +963-11-2131871

Mobile: +963-955-673260

E-mail: raghadn86@hotmail.com

() 2021 Al Nuss and El Zein et al.; Licensee SET Publisher.

This is an open access article licensed under the terms of the Creative Commons Attribution Non-Commercial License (http://creativecommons.org/licenses/by-nc/3.0/) which permits unrestricted, non-commercial use, distribution and reproduction in any medium, provided the work is properly cited. 


\section{INTRODUCTION}

The therapeutic effectiveness of many drugs is often impaired because of their poor bioavailability, mainly due to limited solubility and/or permeability. Lots of drug candidates fail to reach the market because of their poor pharmacokinetics [1]. BCS classification system classifies APIs in four classes based on solubility and permeability [2]. Among them, BCS Class IV drugs suffer from inadequate solubility and permeability, have very poor oral bioavailability, and are inclined to exhibit very large inter-and intrasubject variability [3].

Cefdinir is a third-generation oral cephalosporin antibiotic [4]. It belongs to BCS class IV, with low solubility resulting in low bioavailability of about $21 \%$ for capsules and $25 \%$ for suspension [5]. Its solubility is highly dependent on the $\mathrm{pH}$ of the media $[6,7]$. Therefore, a strategy to enhance solubility/dissolution and ultimately the oral bioavailability of cefdinir would be important to improve the therapeutic effect in the body after oral administration.

Several approaches have been investigated to improve the dissolution rate and bioavailability of Cefdinir, including nanosuspensions [8, 9], amorphization [10], cyclodextrin complexation [7, 11], self-emulsification [12], and solid dispersions (SDs) [13-17].

Among many approaches to improve the dissolution rate of poorly soluble drugs, the use of inorganicorganic hybrid materials has been recognized as one of the most promising systems [18]. This approach has received increased acceptance and awareness concerning its potential use as dissolution enhancers in pharmaceutical formulations by using Mesoporous Silica Materials (MSMs) for the encapsulation of APIs. This type of material possesses a range of controllable properties that can be utilized for the benefit of preserving the loaded drug in its amorphous form within the porous structure, consequently leading to enhanced dissolution behavior. The course of action further results in the protection of the drug molecules from oxidation, hydrolysis, and other degradation processes by restriction of access from the surrounding environment of APIs [19]. MSMs may act as delivery carriers providing a controlled/targeted delivery of drugs and/or to improve their long-term stability and storage by protecting them from unfriendly environments [20]. MSMs are ideal candidates as drug carriers, as silica is a "generally regarded as safe" (GRAS) material, is biodegradable, and can be readily surface-modified to optimize drug loading and subsequent release in the human body. The major advantage of mesoporous silica as drug delivery systems (DDSs) for poorly water-soluble drugs lies in their pore size, pore morphology, and versatility in alteration of the surface groups, which can result in optimized interactions between a drug candidate and MSM carrier by modifying the pore surfaces [19]. According to the method of synthesis and its characteristics, MSN can be synthesized in a variety of forms. MCM-41, developed by Mobil Crystalline Materials or Mobil Composition of Matter, is one of the most widely designed MSNs, with a hexagonal layout and a pore width between 2.5 and $6 \mathrm{~nm}$. Other MSNs from the MCM category are MCM-48 and MCM-50, with cubic and lamellar arrangements, respectively. Subsequently, the Santa Barbara amorphous type materials, including SBA-11 (cubic), SBA-12 (3D hexagonal), SBA-15 (hexagonal), and SBA-16 (cubiccage structures), which have larger pores and thicker silica walls, have been synthesized by the University of California, Santa Barbara [21]. The mesoporous MCM41 silicate was used as a drug carrier for many drugs, and was evaluated as a matrix for improving the dissolution rate of many APIs like Furosemide [20]. But, notwithstanding the positive results in these terms, Furosemide loading was too low for acceptable industrial application. The possible reason may be ascribed to the small diameter of $\mathrm{MCM}-41$ pores if compared to drug molecule size.

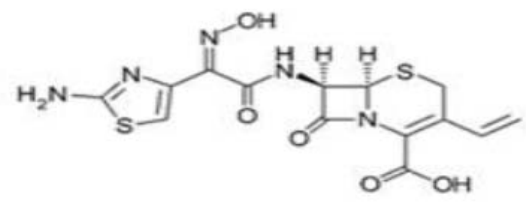

a

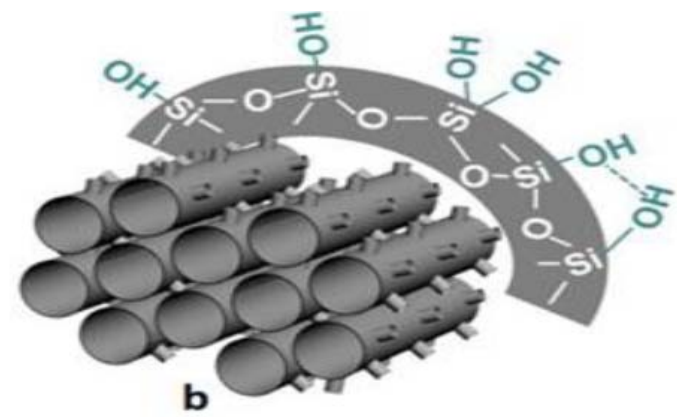

Figure 1: a) the chemical structure of cefdinir, b) the porous structure of SBA-15 and the silanol groups present on the surface. 
In this research, SBA-15 (Figure 1b) has been selected as a carrier for Cefdinir. This type of silica is characterized by larger pores than MCM-41, a high surface constituted by hexagonally ordered tuneable uniform mesopores (4-14 nm) [22], and thick silica pore walls $(3-6 \mathrm{~nm})$ responsible for high hydrothermal stability [23]. Also, SBA-15 has a higher hydrophilic character than MCM-41 due to the higher amount of silanol groups that can interact with many compounds forming hydrogen bonds [24]. For this reason, SBA-15 can adsorb drug molecules by forming weak interactions which can be easily broken in biological fluids allowing a rapid drug release [25].

This work aimed to include Cefdinir into SBA-15 pores to realize a delivery system capable to improve drug dissolution rate and enhancing its physical stability.

\section{MATERIALS AND METHODS}

\subsection{Materials}

Cefdinir was obtained from (Lupin Co.Ltd., India). SBA15 was obtained from (Jiangsu XFNANO Materials Tech Co., Ltd., China). All other used reagents and solvents were of analytical grade and were used as received.

\subsection{Instrumentation}

- Magnetic stirrer (MSH300) from (BOECO, Germany).

- UV-VIS Spectrophotometer (T80) from (PG, United Kingdom).

- Infrared spectrophotometer (FTIR) (Vector 22) from (Bruker, Germany).

- Differential Scanning calorimetry (DSC 131) from (Setaram, France).

- $\quad$ Powder X-ray diffraction device (PXRD) from (Bruker D8, West Germany).

- $\quad$ Oven (Binder $\mathrm{GmbH}$, Germany).

- Dissolution apparatus (PTDT7) from (Pharmatest, Germany).

- Hermle Z 200 A Centrifuge, Germany

- $\quad$ Scanning electron microscopy (SEM, VEGA II LSH, TESCAN, Czech Republic).

\subsection{Solubility Study}

Solubility measurements were performed in triplicate using the shake flask method [26]. Excess cefdinir 100 $\mathrm{mg}$ was added to $10 \mathrm{~mL}$ of each solvent. The samples were subjected to a constant shaking for 72 hours, then centrifuged (5000 x g, 30 min, using Hermle Z 200 A Centrifuge, Germany), filtered (0.22 micron), diluted, and analyzed by UV-VIS spectrophotometer (PG Instruments T80 UVIVIS spectrophotometer, United Kingdom) at $287 \mathrm{~nm}[27,28]$.

\subsection{Loading Cefdinir into SBA-15}

The solvent immersion method was used to load Cefdinir onto the SBA-15. Table 1 shows the solvent used in the loading process for each formula.

Table 1: The Solvent used in the Loading Process for each Formula

\begin{tabular}{|c|c|}
\hline Formulation & Loading solvent \\
\hline \hline F1 & Methanol \\
\hline F2 & Ethanol \\
\hline F3 & $n$ - Hexane \\
\hline F4 & DMSO \\
\hline F5 & DCM \\
\hline
\end{tabular}

Firstly, solutions of cefdinir using different organic solvents were prepared with a concentration depends on drug solubility results in different solvents $(2,1,30$, 30 , and $100 \mathrm{mg} / \mathrm{mL}$ by using methanol, ethanol, $n$ hexane, dichloromethane, and Dimethyl Sulfoxide (DMSO), respectively. Then, SBA-15 particles $(25 \mathrm{mg})$ were added to $25 \mathrm{~mL}$ of cefdinir solution. The ratio between the silica particles and the API solution was kept constant at 1:1000 (w/v) to have an excess amount of API in during the loading process. The sample was kept under stirring for $24 \mathrm{~h}$ at ambient temperature $\left(25^{\circ} \mathrm{C}\right)$. Subsequently, the sample was centrifuged for $30 \mathrm{~min}$ at $8000 \mathrm{rpm}$ to separate the supernatant from the precipitated solid (Cefdinir/SBA15). Then, the materials were dried at room temperature for $72 \mathrm{~h}$. Then, the solids were heated at $60^{\circ} \mathrm{C}$ using an oven (Binder $\mathrm{GmbH}$, Germany) until a constant weight was reached [29].

\subsection{Drug Loading Study}

The loading of cefdinir into SBA-15 was determined by a UV spectrophotometer. Initially, an amount of each 


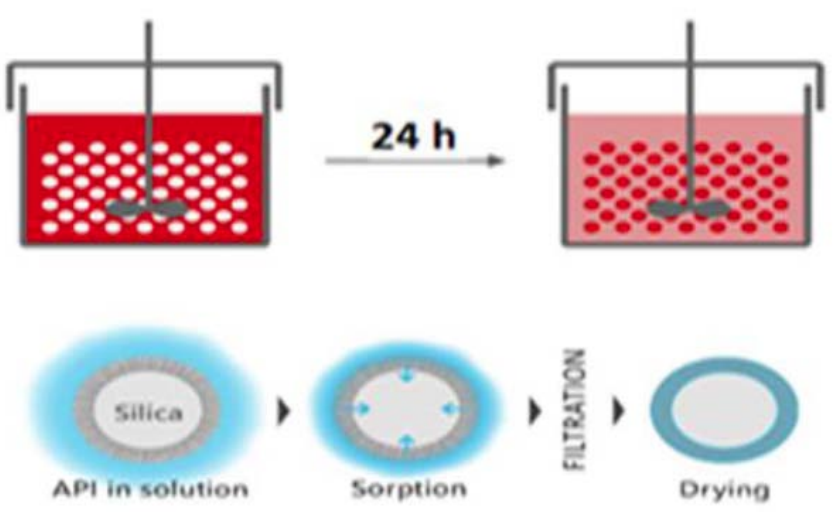

Figure 2: A schematic diagram of the drug loading process through balanced adsorption of the solution.

formulation equivalent to $10 \mathrm{mg}$ drug was dissolved in $10 \mathrm{ml}$ of a phosphate buffer with a $\mathrm{pH}$ value 6.8 , then the samples were kept suspended in the buffer under stirring for an hour, then the solution was diluted appropriately using the same buffer and evaluated by using PG instruments T80 UV/VIS spectrophotometer at $\lambda_{\max } 287 \mathrm{~nm}$. The loading percentage was calculated according to Equation (1) [30].

$\%$ Loading $=\frac{\text { weight of loaded drug }}{\text { weight of } S B A-15} \times 100$

\subsection{Characterization of Cefdinir-Loaded SBA-15}

\subsubsection{Scanning Electron Microscope (SEM)}

The morphology and particle size of pure cefdinir, SBA15, and Cefdinir-loaded silica was observed through scanning electron microscopy (SEM, VEGA II LSH, TESCAN, Czech Republic). Prior to SEM imaging, samples were coated with gold by a sputter coater. Approximately $1 \mathrm{mg}$ of each sample was placed onto a double-sided adhesive strip on a sample holder.

\subsubsection{Fourier Transform Infrared Spectroscopy (FTIR)}

Fourier transform-infrared (FTIR) spectra of samples were recorded using a Bruker Vector 22 spectrophotometer. Samples were compressed into $\mathrm{KBr}$ disks in a hydraulic press to prepare sample- $\mathrm{KBr}$ blends. $\mathrm{KBr}$ pellets were characterized from 400 to $4000 \mathrm{~cm}^{-1}$ and with a resolution of $2 \mathrm{~cm}^{-1}$ [28].

\subsubsection{Differential Scanning Calorimetry (DSC)}

The thermal properties of samples were characterized by DSC instrument DSC 131 (SETARAM, France). Each sample was accurately weighed (equivalently to 5 $\mathrm{mg}$ ) into an aluminum pan and heated in the range of $(30-300)^{\circ} \mathrm{C}$, at a scanning rate of $(10)^{\circ} \mathrm{C} / \mathrm{min}$ under nitrogen airflow of $(50) \mathrm{mL} / \mathrm{min}$ [28].

\subsubsection{Powder X-Ray Diffraction}

Powder X-ray diffraction patterns of cefdinir, and the selected formula (F3), which has been selected because it showed the highest drug loading percentage, were obtained using Bruker's D8 Advance diffractometer (Karlsruhe, West Germany). Powder Xray diffraction (PXRD) patterns were recorded using Germanium monochromatic and $\mathrm{Cu}$ radiation with a nitrogen filter at a voltage of (50) $\mathrm{KeV}$ and a current of (30) $\mathrm{mA}$. The samples were analyzed over the $2 \theta$ range of $(3-60)^{\circ}[28]$.

\subsection{Drug Release Studies}

Dissolution studies were carried out using the USP paddle method at $(37 \pm 0.2)^{\circ} \mathrm{C}$ in a Pharma test PT-DT7 dissolution tester (Germany) at $50 \mathrm{rpm}$ with $900 \mathrm{~mL}$ of $\mathrm{HCl}$ buffer $(\mathrm{pH} 1.2)$, acetate buffer $(\mathrm{pH} 4.5)$ and phosphate buffer $(\mathrm{pH} \mathrm{6.8)} \mathrm{fluids} \mathrm{as} \mathrm{dissolution} \mathrm{media}$ [31]. The selected formula F3 (equivalent to $300 \mathrm{mg}$ of cefdinir) and pure drug were dispersed in the dissolution media, and at predefined intervals, 5-ml samples were withdrawn at $(2,5,10,15,20,30,45$, and 60) minutes, filtered through $0.45 \mu \mathrm{m}$ syringe filter, and assayed via UV-VIS spectrophotometer (T80 PG, United Kingdom) for released drug at $\lambda_{\max }(280,286$, and $287 \mathrm{~nm}$ ) for $\mathrm{HCl}$ buffer $\mathrm{pH}=1.2$, acetate buffer $\mathrm{pH}=4.5$, and phosphate buffer $\mathrm{pH}=6.8$, respectively. An equivalent amount of release medium was supplemented to keep the volume constant. Each experiment was repeated six times at least.

\subsection{Statistical Analysis of the Drug Release Profile}

All the results were expressed as mean values \pm standard deviation. The difference between percentages (fractions) of released cefdinir at each time interval from the pure drug and F3 were statistically evaluated by using a two-tailed Student's ttest. All data analysis was performed using the Microsoft Excel 2019 software. A confidence limit of $p<0.05$ was fixed for the interpretation of the results.

\subsection{Mathematical Modeling of Release Kinetics:}

Cefdinir and Cefdinir-loaded carrier (F3) release kinetics were analyzed by employing the Probit model [32, 33] (Eq.2), the Gompertz model [34] (Eq. 3), the Weibull function [35] (Eq. 4), and the logistic function [36] (Eq. 5). 
$F=F_{\max } \Phi(\alpha+\beta \log t)$

$F=F_{\max } e^{-\alpha e^{\wedge}(-\beta \log t)}$

$F=F_{\max }\left[1-e^{-(t \beta / \alpha)}\right]$

$F=F_{\max }\left[\frac{e^{\wedge}(\alpha+\beta \log t)}{1+e^{\wedge}(\alpha+\beta \log t)}\right]$

where $F$ is the fraction (\%) of drug released in time $t$ and $F_{\max }$ is the maximum fraction of the drug released at infinite time. $\alpha$ is the scale parameter in each model and is related to the time scale of the process. Whereas $\beta$ is the acceleration or shape parameter associated with the dissolution rate per unit of time. In Eq. (2), $\Phi$ is the standard normal distribution of dissolution profile.

In Equations (3-5), the kinetic parameters were obtained after linearization while the parameters in Eq. (1) were adjusted minimizing the sum of squared errors between the experimental and drug released fractions calculated from the cumulative distribution function. Additionally, the accuracy of the obtained parameters was validated employing the DDSolver software [37].

The determination coefficient $\left(r^{2}\right)$ and Akaike Information Criteria (AIC) were used in this study to evaluate the goodness of the release models employed to describe the dissolution profiles [38]. The AIC is defined by equation (6):

$A I C=n \ln (W S S R)+2 p$

where $\mathrm{n}$ is the number of dissolution measurements, $\mathrm{p}$ is the number of parameters that were adjusted in each model, WSSR is the weighted sum of square residues. When comparing mathematical models, a smaller AIC value leads to a better fit.

\subsection{Stability Studies}

The samples of the selected formula (F3) were stored at different conditions of temperature and relative humidity for the study of the physical stability of the amorphous state of loaded Cefdinir: $25^{\circ} \mathrm{C} / \mathrm{RH} 0 \%$, $40^{\circ} \mathrm{C} / \mathrm{RH} 0 \%$ and $40^{\circ} \mathrm{C} / \mathrm{RH} 75 \%$. The samples were first characterized by using PXRD as prepared $(t=0)$, and then some samples were placed in a desiccator with phosphorous pentoxide as a desiccant to produce $0 \%$ relative humidity, and then the desiccator was placed in an oven at two different temperatures $25^{\circ}$, and $40^{\circ} \mathrm{C}$ [39]. The other samples were placed in a desiccator with a saturated salt solution of sodium chloride $(\mathrm{NaCl})$ producing a relative humidity atmosphere of $75 \% \mathrm{RH}$, and then the desiccator was placed in an oven at $40^{\circ} \mathrm{C}$ [20]. The samples were protected from light. The storage period was 6 months, at predetermined intervals ( 1 week, 2 weeks, 1 month, 2 months, 3 months, and 6 months), samples were characterized again to evaluate if drug crystal growth occurred during storage time.

\section{RESULTS AND DISCUSSION}

\subsection{Solubility Results}

Initially, Cefdinir solubility in several organic solvents was studied to select the best solvent for the drug, but it was noticed through the results shown in Table 2 that cefdinir solubility was poor in most of the solvents. As the table shows, cefdinir is slightly soluble in ethanol and methanol, and these results are in agreement with previous studies [40], while cefdinir is soluble in both $n$ hexane, dimethylformamide, and dichloromethane. and freely soluble in dimethyl sulfoxide (DMSO).

Table 2: Solubility Results of Cefdinir in the Different used Organic Solvents

\begin{tabular}{|c|c|}
\hline Solvent & Solubility $(\mathrm{mg} / \mathrm{mL})$ \\
\hline \hline Methanol & $2.076 \pm 0.229$ \\
\hline Ethanol & $1.116 \pm 0.342$ \\
\hline$n$-Hexane & $45.665 \pm 0.003$ \\
\hline DMSO & $390.297 \pm 0.576$ \\
\hline DMF & $35.352 \pm 0.008$ \\
\hline DCM & $55.783 \pm 0.294$ \\
\hline
\end{tabular}

This poor solubility of cefdinir in most organic solvents can be attributed to its hydrophobic properties and low wettability [40].

A key question from the formulation process point of view is solvent selection. Specifically, it is important to know whether a "good" solvent (i.e. solvent in which the API solubility is high and therefore a high concentration can be used during loading) is automatically also a good loading medium. In previously published works where the solvent immersion method was used for drug loading, generally, very high concentrations of well soluble APIs were used [41-43]. Such high concentrations are often not reachable with poorly soluble APIs such as those considered in the present work. 


\subsection{Drug Loading Results}

The concentrations of the loading solutions for each solvent were chosen based on the solubility values, so as to be as high as possible but without risking precipitation of the API. The rationale behind trying to use as high concentrations as possible in each solvent is that the loading method is based on adsorption equilibrium between the solution and the silica particles. Irrespective of the type of the adsorption isotherm (Langmuir, Freundlich, etc.), the equilibrium amount adsorbed is expected to be an increasing function of concentration [29].

Figure 3 shows the results of loading cefdinir using different solvents. Although DMSO is the best solvent for cefdinir, the loading percentage obtained with it was very low $0.23 \%$, this can be attributed to the high polarity of this solvent, and therefore its molecules will compete with drug molecules for bonding with silica particles [44]. Whereas, methanol and ethanol performance was also poor. Indeed, the poor performance of ethanol in loading drugs has been noted in previous work [45]. This is because methanol and ethanol are polar solvents with high solubility parameters of 12.92 and 7.24, respectively [46], Figure 1a shows cefdinir structure which contains an amine group and a carboxyl group that is capable of forming bonds. Therefore, cefdinir will bond with ethanol and methanol with hydrogen bonds, and thus the drug will diffuse more quickly in these solvents and collect in nanocrystals inside silica particles [46]. While the best performance was obtained using $n$-hexane $37 \%$, followed by dichloromethane $21 \%$, because they are non-polar solvents and therefore will not compete with drug molecules for bonding with silica molecules [46].
Thus, it can be said that a good solvent for a particular drug means that the interaction between the solvent molecule and the drug molecule is strongly preferred [47], and thus will compete with the adsorption of the drug on the silica surface. Similarly, although wetting the surface of the silica by the solvent is necessary for adsorption, the very strong interaction between the solvent and silica will also compete with the adsorption of the active substance. The high loading ratio obtained with $n$-hexane can be explained as the less polar solvent and therefore will not compete with highly hydrophobic materials such as cefdinir for adsorbing it on the silica surface, which led to a significant increase in the loading ratio [48].

\subsection{Scanning Electron Microscope (SEM)}

SEM was used to compare the morphology and particle size of pure cefdinir, SBA-15, and cefdinir-loaded SBA15. SEM micrographs of pure cefdinir, SBA-15, and drug-loaded SBA-15 are shown in (Figure 4). Pure drug consisted of an acicular shaped particle with a mean particle size $(1.224 \pm 0.619) \mu \mathrm{m}$. SBA-15 (Figure 4b) consisted of arrangements of rod-shaped particles attached with a uniform grain size of approximately 1 $\mu \mathrm{m}$, resembling the structure of a rope. Whereas, Figure 4c shows drug-loaded SBA-15, as the figure shows that the original morphology of cefdinir disappeared which may indicate that cefdinir was completely loaded into silica.

\subsection{Fourier Transform Infrared Spectroscopy (FTIR)}

Figure 5 shows the FTIR spectra of the pure drug, SBA-15, and the prepared formula that gave the highest loading percentage (F3).

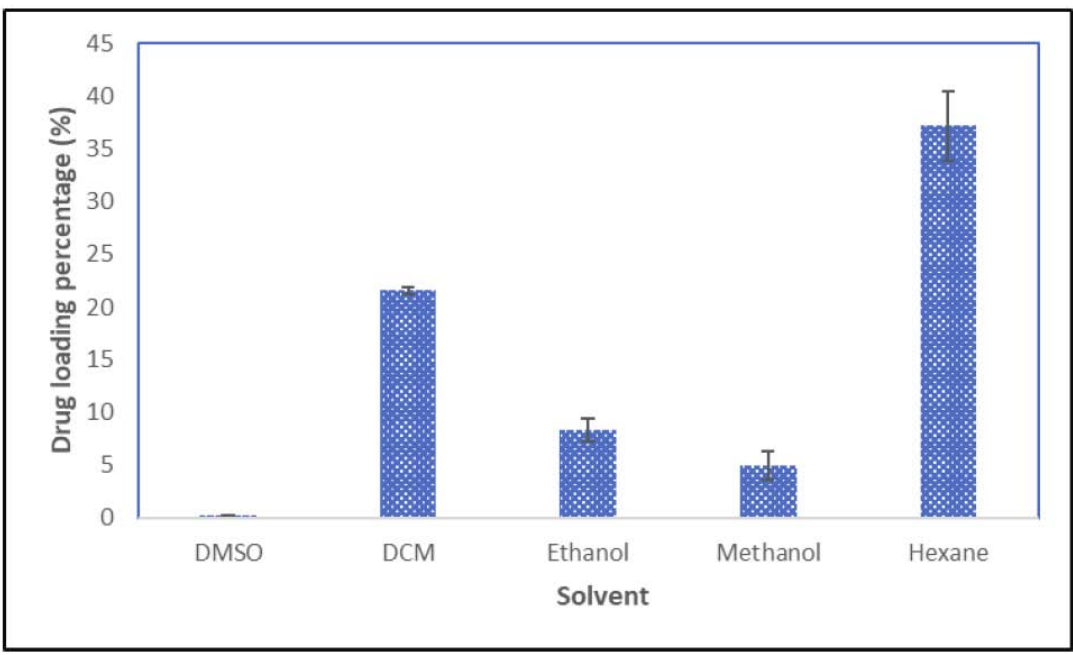

Figure 3: Results of loading cefdinir using different solvents. 

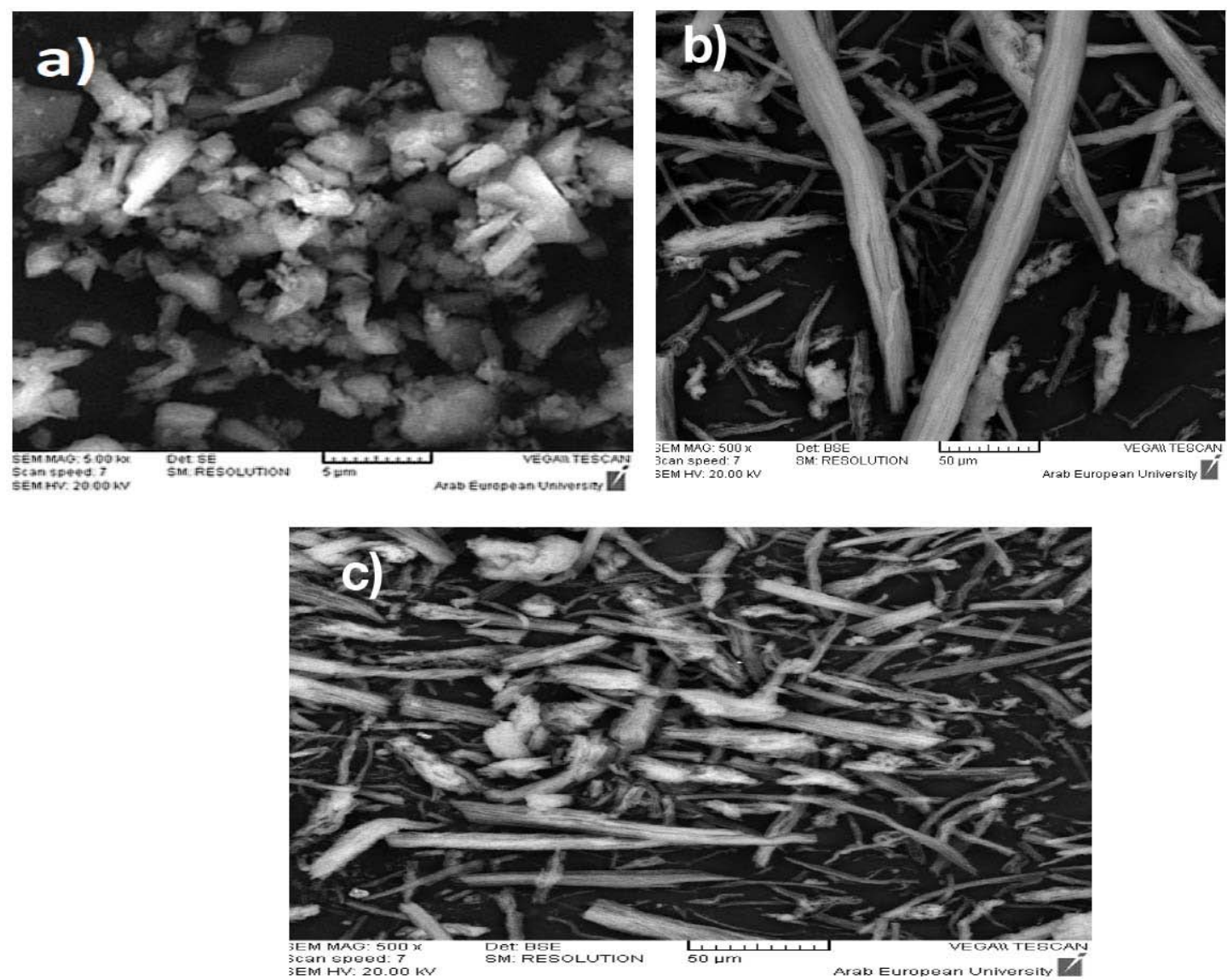

Figure 4: SEM results for: a) pure cefdinir X 10,000, b) SBA-15X 1000, c) SBA-15 loaded with cefdinir X 1000.

The FTIR spectrum of cefdinir (5a) is characterized by the main absorption values at $3300 \mathrm{~cm}^{-1} \mathrm{OH}$ stretching of the carboxyl group, $2978 \mathrm{~cm}^{-1}$ cyclic $\mathrm{CH}$ stretch, $2898 \mathrm{~cm}^{-1} \mathrm{CH}$ stretching, $1781 \mathrm{~cm}^{-1}$ (CO), $1763 \mathrm{~cm}^{-1}$ (CO of $\beta$-lactam), 1710 and $1735 \mathrm{~cm}^{-1}$ carbonyl stretch peaks of carboxylate function, $1667 \mathrm{~cm}^{-1}$ (CC alkene), $1610 \mathrm{~cm}^{-1}$ (aromatic CC), $1544 \mathrm{~cm}^{-1} \mathrm{NH}$ curvature, $1428 \mathrm{~cm}^{-1}$ stretch of the $\mathrm{CN}, 1352 \mathrm{~cm}^{-1}$ for $\mathrm{NH}_{2}$ and 625 $\mathrm{cm}^{-1}$ for CS [7].

The FTIR spectrum of SBA-15 (Figure 5 b) shows a vibrational band at $3744 \mathrm{~cm}^{-1}$ (which is attributed to isolated peripheral silanol groups) and a very wide band between 3200 and $3600 \mathrm{~cm}^{-1}$ that is attributed to hydrogen-bonded silanol groups [25]. While the spectrum of the selected formula shows several changes which are: (1) decrease in the intensity of the vibration beam at $3744 \mathrm{~cm}^{-1}$; (2) A large absorption band between 2900 and $3600 \mathrm{~cm}^{-1}$ is due to the formation of hydrogen bonds between the drug molecule and the silanol groups, (3) the absence of an absorption band at $1352 \mathrm{~cm}^{-1}$ that belongs to the $\mathrm{NH}_{2}$ of the drug. These results could indicate the formation of hydrogen bonds between the cefdinir and the terminal silanol groups [25].

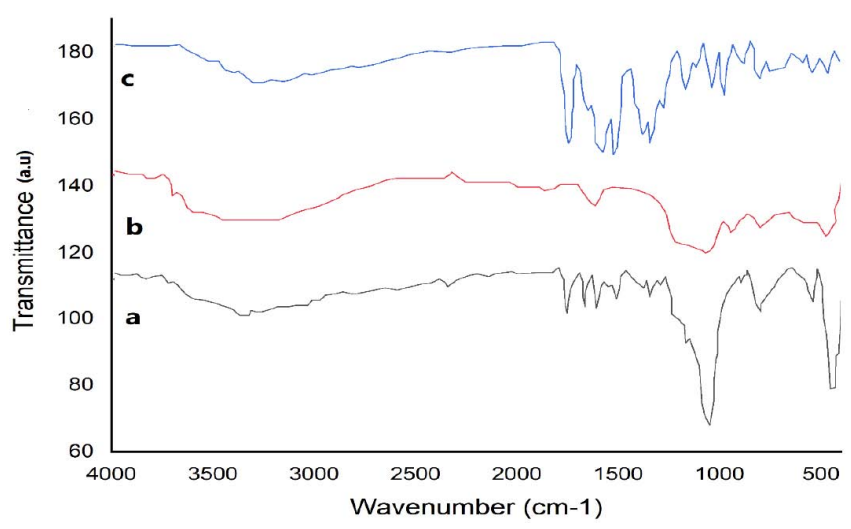

Figure 5: FTIR results for: a) pure cefdinir, b) SBA-15, c) SBA-15 loaded with cefdinir.

\subsection{Differential Scanning Calorimetry (DSC)}

Figure $6 \mathbf{a}$ shows the thermogram of pure cefdinir, where two peaks are observed: the first is a small endothermic peak at $64.5^{\circ} \mathrm{C}$ indicates the glass transition temperature of the drug [49], and the other is a sharp endothermic peak at $229.52^{\circ} \mathrm{C}$ that represents the drug's degradation upon melting [50]. These results were in good agreement with the reported DSC thermogram of cefdinir anhydrous [50]. 

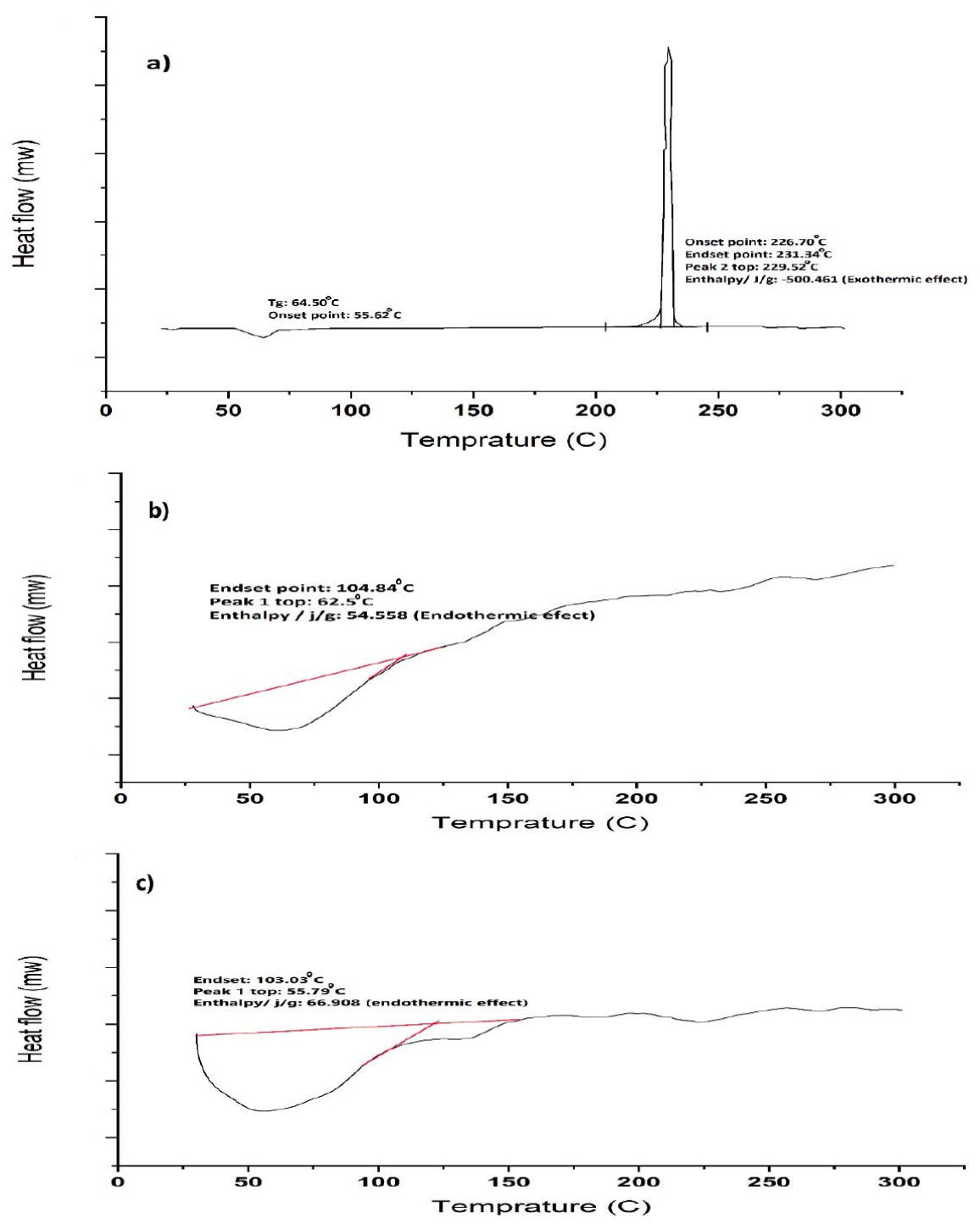

Figure 6: DSC thermograms for: a) cefdinir, b) SBA-15, c) drug loaded silica.

Figure $\mathbf{6 b}$ shows the thermogram of SBA-15 which shows the presence of a broad endothermic peak at $62.5^{\circ} \mathrm{C}$ that can be attributed to the gradual release of water from the sample because silica has hygroscopic properties [51]. Whereas, the thermogram of the selected formulation (F3) shows the absence of the melting peak of cefdinir, which may indicate the transformation of the drug to the amorphous form upon adsorption on the outer surface of mesoporous silica [51].

\subsection{Drug Release Studies}

The dissolution studies for pure cefdinir and the selected formulation (F3) were performed in $\mathrm{HCl}$ buffer $(\mathrm{pH}$ 1.2), acetate buffer ( $\mathrm{pH} 4.5)$, and phosphate buffer ( $\mathrm{pH}$ 6.8) fluids. As shown in Figure 7, Cefdinir (pure drug) and $\mathrm{F} 3$ showed relatively higher dissolution at $\mathrm{pH}$
6.8 (Figure 7c) compared to $\mathrm{pH} 1.2$ and $\mathrm{pH} 4.5$ (Figure $7 \mathrm{a}, 7 \mathrm{~b}$ ), due to the poor solubility of cefdinir at low $\mathrm{pH}$. And, this can be explained by the fact that Cefdinir contains three ionizable groups: the $\mathrm{COOH}$ carboxyl group of the cepheme moiety pKa $=1.9$, the $\mathrm{NH}_{2}$ group of the aminothiazole moiety $\mathrm{pKa}=3.3$, and the $\mathrm{N}-\mathrm{OH}$ group of the oxime moiety $\mathrm{pKa}=9.9$. Thus, according to the Henderson-Hasselbach equation, the carboxyl group will almost completely ionize above $\mathrm{pH} 4.5$, which explains the higher dissolution of the drug in the phosphate buffer compared to the rest of the media [52].

Initially in hydrochloric acid buffer, it was observed that about $65 \%$ of cefdinir was released from F3 within 5 minutes compared to $40 \%$ in the case of the crystalline drug, and the release from $\mathrm{F} 3$ reached $99 \%$ after 20 minutes, while the release of the pure drug was only 

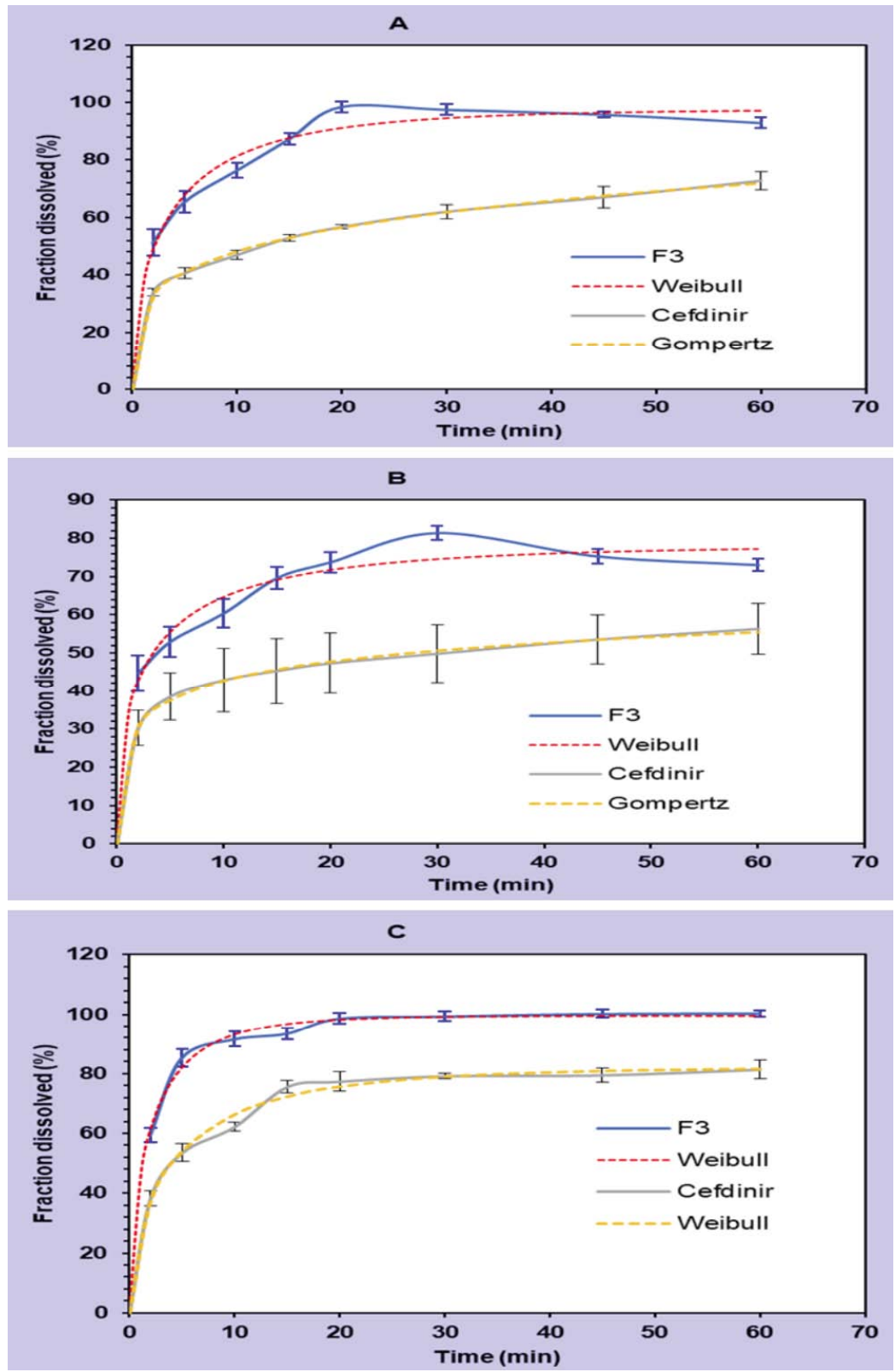

Figure 7: Cefdinir release profiles in A) hydrochloric acid buffer, B) acetate buffer, C) phosphate buffer.

$72 \%$ at the end of the test (that is, after 60 minutes), and there was a statistically significant difference at all studied time points at the level of significance $(P<0.05)$.

The enhancement of drug dissolution observed for the inclusion product SBA-15-Cefdinir could be explained through four different steps [53]: (i) the imbibition of release medium into the system driven by osmotic pressure arising from concentration gradients; (ii) drug dissolution; (iii) drug diffusion through the continuous matrices due to concentration gradients; (iv) drug diffusional and convective transport within the release medium. It can be hypothesized that the main mechanism governing Cefdinir release from SBA-15 loaded product was the rapid matrix imbibition that promoted faster drug dissolution in comparison to pure drug. In this way, after contacting Gl fluids, a drug supersaturated solution is produced [54] improving drug diffusion in the dissolution medium.

Moreover, the high concentration of drug obtained after few minutes is attributable both to the rapid matrix imbibition and to a consequent immediate formation of bindings among water molecules and matrix silanols. The ability of the matrix to create a supersaturation condition is an interesting property influencing drug absorption since, according to Fick diffusion law, high differences of drug concentration between the apical and basolateral sides of gastric mucosa enhance drug diffusion across it [25]. 
Table 3: Cefdinir, Cefdinir/SBA-15 (F3) Kinetics Parameters According to Probit, Gompertz, Weibull and Logistic Models

\begin{tabular}{|c|c|c|c|c|c|c|c|}
\hline \multirow[t]{2}{*}{ Model } & \multirow[t]{2}{*}{ Statistics } & \multicolumn{2}{|c|}{$\mathrm{HCl}$ buffer } & \multicolumn{2}{|c|}{ Acetate buffer } & \multicolumn{2}{|c|}{ Phosphate buffer } \\
\hline & & Cefdinir & F3 & Cefdinir & F3 & Cefdinir & F3 \\
\hline \multirow[t]{4}{*}{ Probit } & $\alpha$ & -2.483 & -0.438 & -1.082 & -0.279 & -0.567 & -0.269 \\
\hline & $\beta$ & 0.203 & 1.303 & 0.497 & 1.090 & 1.335 & 1.736 \\
\hline & $r^{2}$ & 0.994 & 0.930 & 0.991 & 0.896 & 0.974 & 0.987 \\
\hline & $\mathrm{AIC}$ & 16.428 & 45.957 & 14.685 & 43.898 & 36.365 & 28.938 \\
\hline \multirow[t]{4}{*}{ Gompertz } & $\alpha$ & 5.913 & 1.232 & 2.792 & 1.047 & 1.411 & 1.143 \\
\hline & $\beta$ & 0.101 & 1.459 & 0.470 & 1.187 & 1.462 & 2.539 \\
\hline & $r^{2}$ & 0.998 & 0.920 & 0.994 & 0.899 & 0.969 & 0.980 \\
\hline & $\mathrm{AIC}$ & 14.418 & 46.949 & 14.238 & 44.959 & 37.75 & 32.695 \\
\hline \multirow[t]{4}{*}{ Weibull } & $\alpha$ & 7.062 & 2.230 & 1.937 & 1.860 & 2.556 & 1.684 \\
\hline & $\beta$ & 0.258 & 0.598 & 0.337 & 0.509 & 0.624 & 0.667 \\
\hline & $r^{2}$ & 0.996 & 0.942 & 0.993 & 0.920 & 0.982 & 0.991 \\
\hline & $\mathrm{AIC}$ & 17.281 & 44.302 & 15.651 & 43.140 & 33.442 & 26.645 \\
\hline \multirow[t]{4}{*}{ Logistic } & $\alpha$ & -4.236 & -0.726 & -2.133 & -0.484 & -0.934 & -0.521 \\
\hline & $\beta$ & 0.558 & 1.993 & 0.843 & 1.644 & 2.059 & 2.973 \\
\hline & $r^{2}$ & 0.996 & 0.927 & 0.994 & 0.906 & 0.973 & 0.990 \\
\hline & $\mathrm{AIC}$ & 14.481 & 46.276 & 15.041 & 44.45 & 36.667 & 27.089 \\
\hline
\end{tabular}

\subsection{Mathematical Modeling of Release Kinetics}

The cefdinir release curves from the pure drug and the optimal formula (F3) were evaluated by fitting the experimental data with the Probit, Gompertz, Weibull, and Logistic models. The parameters of each of the kinetics equations, determination coefficient $\left(r^{2}\right)$, and (AIC) are listed in the Table 3 and the results are represented in Figure 7. These values were used to evaluate the goodness of the release models.

Based on the $\left(r^{2}\right)$ and $(A I C)$, it is noted that the Gompertz model is the best function for describing drug release kinetics from pure cefdinir, while the Weibull model is more suitable for the $\mathrm{F} 3$ formula in hydrochloric buffer $\mathrm{pH}$ 1.2. Similar results were also observed in acetate buffer, the Gompertz model was the most suitable for describing drug release kinetics from the pure drug, while the Weibull model was the most suitable for describing the release kinetics from formula F3. While in phosphate buffer, different results were observed, i.e. drug release from cefdinir and the F3 formula was more suitable for the Weibull model.

This can be explained by the fact that the Gompertz model is more useful for comparing the release profiles of drugs having an intermediate release rate as Cefdinir at $\mathrm{pH}$ less than 4.5. This model has a sharp increment in the beginning and converges slowly to the asymptotic maximal dissolution [55]. On the other hand, the Weibull model is more appropriate for comparing the release profiles of drugs loaded into carriers, which significantly increases the drug dissolution rate. In addition, cefdinir has a higher solubility at $\mathrm{pH}$ higher than 4.5 [52]. Figure 7 shows the models that gave the best representation of drug release kinetics in the studied media, to prove the quality and suitability of these parameters.

\subsection{Powder X-Ray Diffraction}

The PXRD pattern of pure cefdinir (Figure 8) showed characteristic peaks at $5.85^{\circ}, 11.7^{\circ}, 16.1^{\circ}, 21.15^{\circ}$, $22.25^{\circ}, 24.4^{\circ}, 26.2^{\circ}$, and $28.8^{\circ}$ of $2 \theta$ [7]. This figure also shows the PXRD of the optimal formula (F3) immediately after preparation, where it was noted that all the characteristic peaks of the drug were absent, which may indicate the drug's transformation to the amorphous form upon adsorption on the silica [20].

\subsection{Stability Study}

Figure 8 shows PXRD of the prepared formulation F3 at the studied time intervals of stability tests using different conditions of temperature and relative humidity, as follows: Figure $8 \mathrm{a}$ Temperature $25^{\circ} \mathrm{C} /$ 


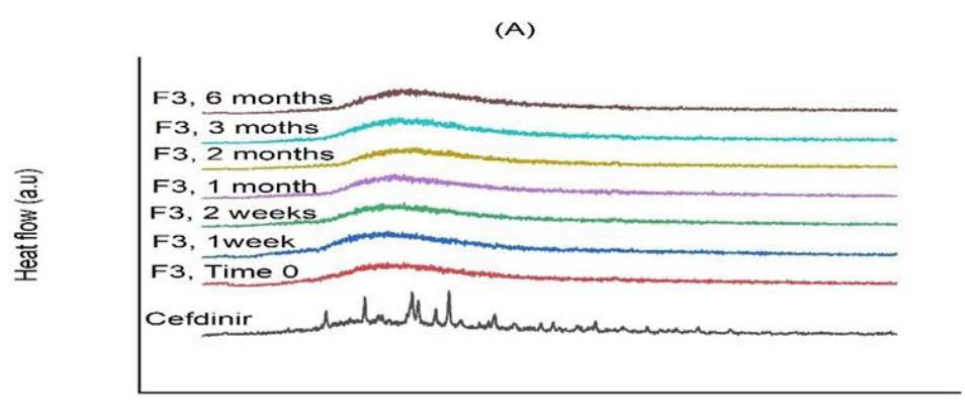

Diffraction angle 2 theta

(B)

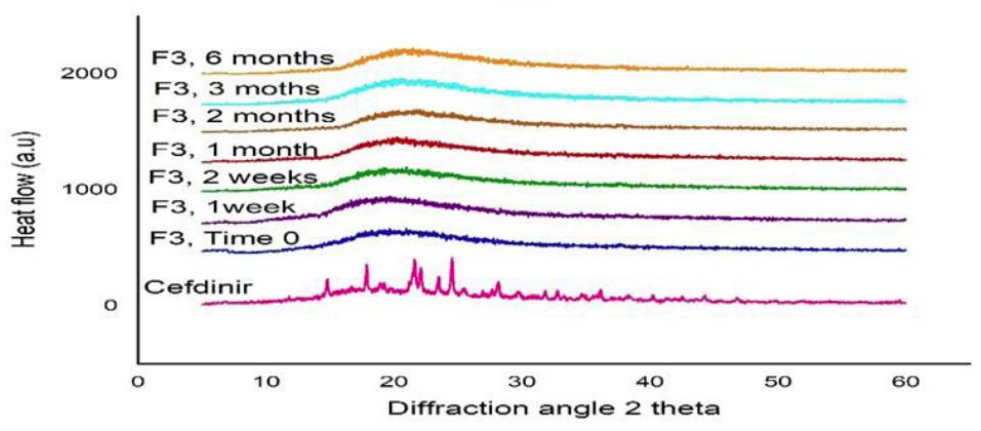

(C)

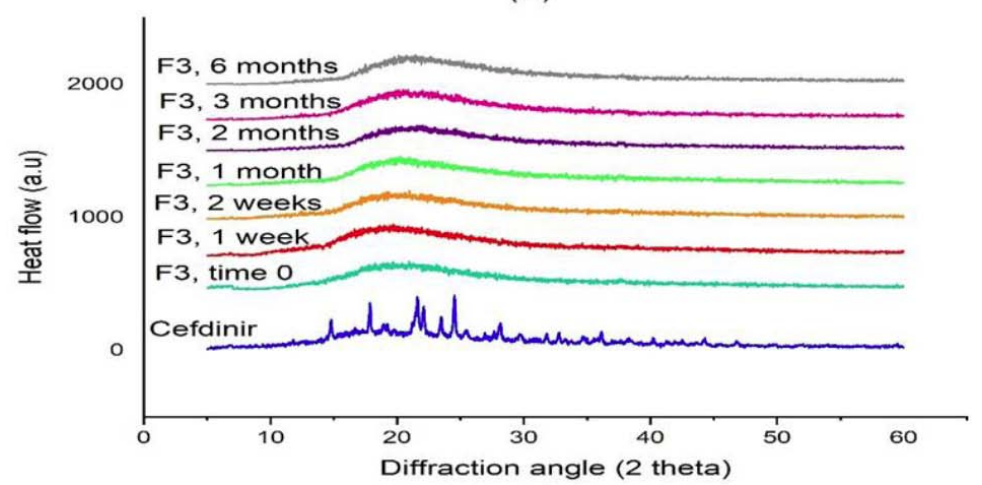

Figure 8: Powder X-ray diffraction plots of cefdinir and $\mathrm{F} 3$ formula over the stability test period at: $(\mathbf{A}) 25^{\circ} \mathrm{C} / 0 \% \mathrm{RH},(\mathrm{B})$ $40^{\circ} \mathrm{C} / 0 \% \mathrm{RH}$, and $(\mathrm{C}) 40^{\circ} \mathrm{C} / \mathrm{RH} 75 \%$ relative humidity.

$0 \%$ relative humidity, Figure $\mathbf{8 b}$ has a temperature of $40^{\circ} \mathrm{C} / 0 \%$ relative humidity, and Figure $8 \mathrm{c}$ has a temperature of $40^{\circ} \mathrm{C} / 75 \%$ relative humidity. During the period of stability studies under different conditions, no evidence of drug crystals was detected as shown in the PXRD charts (Figure 8). This stability can be attributed to the retention of cefdinir within the pores of the silica [56], in addition to the hydrogen bonds formed between the drug and the silanol groups of silica which were detected by FTIR results, these bonds would impede the movement of drug molecules and prevents rearrangement and thus will preserve the amorphous form of the drug [56-58]. These results are in agreement with the results of previous studies conducted on several drugs, such as the study on forsemide, which was loaded on SBA-15, and this study showed that furosemide remained in its amorphous form throughout the stability test period [25].

\section{CONCLUSION}

In this paper, cefdinir, an antibiotic that belongs to BSC class IV, was loaded onto mesoporous silica (SBA-15) in an attempt to overcome the problem of its poor bioavailability and to enhance the physical stability. Different organic solvents were used to complete the loading process, and these solvents varied in performance, the best of which was $n$-hexane, which is a nonpolar solvent and therefore will not compete with drug molecules on adsorption on silica particles. The optimal formula (F3) showed a significant improvement in the dissolution of the drug in the studied media, and succeeded in maintaining the adsorbed cefdinir in its amorphous form when subjected to accelerated 
stability studies using different conditions of temperature and relative humidity. FTIR results revealed a hydrogen bond that formed between the drug and silanol groups of SBA-15. Whereas, DSC and PXRD results showed that cefdinir has been transformed to an amorphous form, and remained amorphous in the optimized formula (F3). Thus, drug adsorption onto mesoporous silica constitutes a promising method in improving the solubility of poorly water-soluble drugs and increasing their physical stability, as is the case for cefdinir.

\section{ACKNOWLEDGEMENT}

R. Al-Nuss acknowledges Diamond pharmaceutical industry for providing research materials. The work was carried out in the Faculty of Pharmacy (Damascus university) and was supported by Atomic Energy Authority (Syria).

\section{FUNDING}

R. Al-Nuss acknowledges Damascus university for funding this research.

\section{AUTHORS' CONTRIBUTION}

The author confirms sole responsibility for the following: study conception and design, data collection, analysis and interpretation of results, and manuscript preparation.

\section{CONFLICT OF INTEREST}

The authors declare that they have no conflict of interest.

\section{STATEMENT OF HUMAN AND ANIMAL RIGHTS}

This article does not contain any studies with human and animal subjects performed by any of the authors.

\section{LIST OF ABBREVIATIONS}

$$
\begin{aligned}
& \text { AIC } \quad \text { Akaike Information Criteria } \\
& \text { BSC } \quad=\text { Biopharmaceutical Classification System } \\
& \text { DMSO = Dimethyl Sulfoxide } \\
& \text { DSC } \quad=\text { Differential Scanning Calorimetry } \\
& \text { FTIR } \quad=\text { Fourier-Transform Infrared Spectroscopy } \\
& \text { MCM-41 = Mobil Composition of Mater No.41 }
\end{aligned}
$$

MSMs = Mesoporous Silica Materials

PXDR = Powder X-Ray Diffraction

$\mathrm{RH} \quad=$ Relative Humidity

SEM = Scanning Electron Microscope

SBA-15 = Santa Barbara Amorphous -15

\section{REFERENCES}

[1] Leuner C, Dressman J. Improving drug solubility for oral delivery using solid dispersions. Eur $\mathrm{J}$ Pharm Biopharm 2000; 50(1): 47-60.

https://doi.org/10.1016/S0939-6411(00)00076-X

[2] Amidon CJ, Lennernas GL, Shah H. A theoreical basis for a Biopharamcetics Drug Classification: The correlation of in vitro drug product dissolution and in vivo bioavailability. Pharm Res 1995; 12(3): 413-20. https://doi.org/10.1023/A:1016212804288

[3] Markovic M, Zur M, Ragatsky H, Cvijić S, Dahan A. Bcs class iv oral drugs and absorption windows: Regional-dependent intestinal permeability of furosemide. Pharmaceutics 2020; 12(12): 1-16.

https://doi.org/10.3390/pharmaceutics12121175

[4] Wise R, Andrews JM, Thbornber D. The in-vitro activity of cefdinir (FK482), a new oral cephalosporin. J Antimicrob Chemother 1991; 28(2): 239-48. https://doi.org/10.1093/jac/28.2.239

[5] Guay DRP. Cefdinir: An advanced-generation, broadspectrum oral cephalosporin. Clin Ther 2002; 24(4): 473-89. https://doi.org/10.1016/S0149-2918(02)85125-6

[6] Perry CM, Scott LJ. Cefdinir: a review of its use in the management of mild-to-moderate bacterial infections. Drugs 2004; 64(13): 1433-64.

https://doi.org/10.2165/00003495-200464130-00004

[7] Aleem O, Kuchekar B, Pore $Y$, Late S. Effect of $\beta$ cyclodextrin and hydroxypropyl $\beta$-cyclodextrin complexation on physicochemical properties and antimicrobial activity of cefdinir. J Pharm Biomed Anal 2008; 47(3): 535-40. https://doi.org/10.1016/j.jpba.2008.02.006

[8] Sawant KK, Patel MH, Patel K. Cefdinir nanosuspension for improved oral bioavailability by media milling technique: formulation, characterization and in vitro-in vivo evaluations. Drug Dev Ind Pharm 2015; 1-11. https://doi.org/10.3109/03639045.2015.1104344

[9] Patil OA, et al. Formulation optimization and evaluation of Cefdinir nanosuspension using 23 Factorial design. Marmara Pharm J 2018; 22(4): 587-598. https://doi.org/10.12991/irp.2018.101

[10] Park J, et al. Preparation and pharmaceutical characterization of amorphous cefdinir using spray-drying and SAS-process. Int J Pharm 2010; 396: 239-45. https://doi.org/10.1016/j.ijpharm.2010.06.032

[11] Morina D, Sessevmez M, Sinani G, Mülazımoğlu L, Cevher E. Oral tablet formulations containing cyclodextrin complexes of poorly water soluble cefdinir to enhance its bioavailability. J Drug Deliv Sci Technol 2020; 57: 101742.

https://doi.org/10.1016/j.jddst.2020.101742

[12] Chhayani RL, Chhayani RB, Patel D, Borkhataria $\mathrm{CH}$. Development and Characterization of Self- Microemulsifying Drug Delivery System for Improvement of Bioavailability of Cefdinir. JPMC 2016; 2(1): 57-83.

https://doi.org/10.21088/jpmc.2395.6615.2116.7 
[13] Cho $\mathrm{HJ}$, et al. Cefdinir Solid Dispersion composed of Hydrophilic Polymers with Enhanced Solubility, Dissolution, and Bioavailability in Rats. Molecules 2017; 22(280): 1-14. https://doi.org/10.3390/molecules22020280

[14] Jain S, Jain S, Mishra A, Garg G, Modi RK. Formulation and characterization of fast disintegrating tablets containing Cefdinir solid dispersion. Int J Pharm Life Sci 2012; 3(12): 2190-99.

[15] Maraie NK, Alhamdany AT, Radhi AA. Efficacy of compenation solid dispersion technology on dissolution performance of nalidixic acid and cefdinir. Asian J Pharm Clin Res 2017; 10(4): 394-401. https://doi.org/10.22159/ajpcr.2017.v10i4.16913

[16] Prathibha B, Reddy DV, Reddy DS. Formulation and evalution of once a day tablet of cefdinir. Int J Pharm Technol 2014; 5(4): 115-6130.

[17] Lakshmanarao $P$, et al. Enhancement of solubility and dissolution rate of cefdinir using solid dispersions. Indo Am J Pharm Res 2017; 4(3): 747-753.

[18] Sharma S, Sher P, Badve S, Pawar AP. Absorption of Meloxicam on Porous Calcium Silicate: Characterization and tablet formulation. AAPS PharmSciTech 2005; 6(4): 618-25. https://doi.org/10.1208/pt060476

[19] Maleki A, Kettiger H, Schoubben A, Rosenholm JM, Ambrogi V, Hamidi M. Mesoporous silica materials: From physicochemical properties to enhanced dissolution of poorly watersoluble drugs. J Control Release 2017; 262: 329-47. https://doi.org/10.1016/j.jconrel.2017.07.047

[20] Ambrogi V, et al. MCM-41 for furosemide dissolution improvement. Micropor Mesopor Mat 2012; 147(1): 343-49. https://doi.org/10.1016/j.micromeso.2011.07.007

[21] Chircov C, et al. Mesoporous Silica Platforms with Potential Applications in Release and Adsorption of Active Agents. Molecules 2020; 25(17): 1-35.

https://doi.org/10.3390/molecules25173814

[22] Celer EP, Kruk M, Zuzek Y, Jaroniec M. Hydrothermal stability of SBA-15 and related ordered mesoporous silicas with plugged pores. J Mater Chem 2006; 16(27): 2824-33. https://doi.org/10.1039/b603723b

[23] Cassiers K, et al. A detailed study of thermal, hydrothermal, and mechanical stabilities of a wide range of surfactant assembled mesoporous silicas. Chem Mater 2002; 14(5): 2317-24.

https://doi.org/10.1021/cm0112892

[24] Ma Y, Qi L, Ma J, Wu Y, Liu O, Cheng H. Large-pore mesoporous silica spheres: Synthesis and application in HPLC. Colloids Surfaces A Physicochem. Eng Asp 2003; 229(1-3): 1-8.

https://doi.org/10.1016/j.colsurfa.2003.08.010

[25] Ambrogi $V$, et al. Use of SBA-15 for furosemide oral delivery enhancement. Eur J Pharm Sci 2012; 46(1-2): 43-48.

https://doi.org/10.1016/j.ejps.2012.02.004

[26] Baka E, Comer JEA, Takács-Novák K. Stuy of equilibrium solubility measurement by saturation shake-flask method using hydrochlorothiazide as model compound. J Pharm Biomed Anal 2008; 46(2): 335-41.

https://doi.org/10.1016/j.jpba.2007.10.030

[27] Shah PB, Pundarikakshudu K. UV spectroscopic and colorimetric methods for the estimation of cefdinir in capsule dosage forms. Indian J Pharm Sci 2004; 66: 665-67.

[28] Al-Badr AA, Alasseiri FA. Cefdinir. In: Profiles of Drug Substances, Excipients and Related Methodology Elsevier Inc. 2014; 39: 41-112. https://doi.org/10.1016/B978-0-12-800173-8.00002-7

[29] Šoltys $M$, Kovačík $P$, Dammer $O$, Beránek J, Š těpánek $F$. Effect of solvent selection on drug loading and amorphisation in mesoporous silica particles. Int J Pharm 2019; 555: 19-27. https://doi.org/10.1016/j.ijpharm.2018.10.075
[30] Albayati TM, Salih IK, Alazzawi HF. Synthesis and characterization of a modified surface of SBA-15 mesoporous silica for a chloramphenicol drug delivery system. Heliyon 2019; 5(10): e02539.

https://doi.org/10.1016/j.heliyon.2019.e02539

[31] Lakshmanarao P, Prasada Rao M, Krishnaveni T, Sukanya B, Lakshmi Priya P, Sai Krishna A. Enhancement of solubility and dissolution rate of cefdinir using solid dispersions. Indo Am J Pharm Sci 2017; 4: 747-53.

[32] Tsong $Y$, Hammerstrom T, Chen JJ. Multipoint dissolution specification and acceptance sampling rule based on profile modeling and principal component analysis. J Biopharm Stat 1997; 7(3): 423-39.

https://doi.org/10.1080/10543409708835198

[33] Shah VP, Tsong Y, Sathe P, Liu JP. In vitro dissolution profile comparison- Statistics and analysis of the similarity factor, f2. Pharm Res 1998; 15(6): 889-96. https://doi.org/10.1023/A:1011976615750

[34] Costa FO, Sousa JJS, Pais AACC, Formosinho SJ. Comparison of dissolution profiles of Ibuprofen pellets. J Control Release 2003; 89(2): 199-12.

https://doi.org/10.1016/S0168-3659(03)00033-6

[35] Langenbucher F. Letters to the Editor: Linearization of dissolution rate curves by the Weibull distribution. J Pharm Pharmacol 1972; 24(12): 979-81. https://doi.org/10.1111/j.2042-7158.1972.tb08930.x

[36] Costa LJ. Modeling and comparison of dissolution profiles. Eur J Pharm Sci 2001; 13: 123-33. https://doi.org/10.1016/S0928-0987(01)00095-1

[37] Zhang Yet al. DDSolver: An add-in program for modeling and comparison of drug dissolution profiles. AAPS J 2010; 12(3): 263-71. https://doi.org/10.1208/s12248-010-9185-1

[38] Simionato LD, Petrone L, Baldut M, Bonafede SL, Segall AI. Comparison between the dissolution profiles of nine meloxicam tablet brands commercially available in Buenos Aires, Argentina. Saudi Pharm J 2018; 26(4): 578-84. https://doi.org/10.1016/j.jsps.2018.01.015

[39] Mellaerts Ret al. Aging behavior of pharmaceutical formulations of itraconazole on SBA-15 ordered mesoporous silica carrier material. Micropor Mesopor Mat 2010; 130(1-3): 154-61. https://doi.org/10.1016/j.micromeso.2009.10.026

[40] Belgic M. US 2013/0017156 A1.

[41] Heikkilä Tet al. Evaluation of mesoporous TCPSi, MCM-41, SBA-15, and TUD-1 materials as API carriers for oral drug delivery. Drug Deliv 2007; 14(6): 337-47. https://doi.org/10.1080/10717540601098823

[42] Hong $S$ et al. High drug load, stable, manufacturable and bioavailable fenofibrate formulations in mesoporous silica: A comparison of spray drying versus solvent impregnation methods. Drug Deliv 2016; 23(1): 316-27. https://doi.org/10.3109/10717544.2014.913323

[43] Salonen $\mathrm{J}$ et al. Mesoporous silicon microparticles for oral drug delivery: Loading and release of five model drugs. J Control Release 2005; 108(2-3): 362-74.

https://doi.org/10.1016/j.jconrel.2005.08.017

[44] Chaudhari SP, Gupte A. Mesoporous silica as a carrier for amorphous solid dispersion. Br J Pharm Res 2017; 16(6): 119.

https://doi.org/10.9734/BJPR/2017/33553

[45] Hillerström A, Andersson M, Samuelsson J, Van Stam J. Solvent strategies for loading and release in mesoporous silica. Colloid Interface Sci Commun 2014; 3: 5-8. https://doi.org/10.1016/j.colcom.2015.01.001

[46] Eren ZS, Tunçer S, Gezer G, Yildirim LT, Banerjee S, Yilmaz A. Improved solubility of celecoxib by inclusion in SBA-15 
mesoporous silica: Drug loading in different solvents and release. Micropor Mesopor Mat 2016; 235: 211-23.

https://doi.org/10.1016/j.micromeso.2016.08.014

[47] Tomasi J.Thirty years of continuum solvation chemistry: A review, and prospects for the near future. Theor Chem Acc 2004; 112: 184-03.

https://doi.org/10.1007/s00214-004-0582-3

[48] Fang R, Yang S, Wang Y, Qian H. Nanoscale Drug Delivery Systems: A Current Review on the Promising Paclitaxel Formulations for Future Cancer Therapy. Nano 2015; 10(5): 1-27.

https://doi.org/10.1142/S1793292015300042

[49] Server NE, and Law D. WO2005100368A2. 2006.

[50] Cabri W, et al. Cefdinir: A comparative study of anhydrous vs. monohydrate form. Microstructure and tabletting behaviour. Eur J Pharm Biopharm 2006; 64(2): 212-21. https://doi.org/10.1016/j.ejpb.2006.05.007

[51] Kiwilsza A, Mielcarek J, Pajzderska A, Waosicki J. Ordered mesoporous silica material SBA-15: Loading of new calcium channel blocker-lacidipine. J Microencapsul 2013; 30(1): 2127. https://doi.org/10.3109/02652048.2012.692401

[52] Lepsy CS, Guttendorf RJ, Kugler AR, Smith DE. Effects of organic anion, organic cation, and dipeptide transport inhibitors on cefdinir in the isolated perfused rat kidney. Antimicrob Agents Chemother 2003; 47(2): 689-96. https://doi.org/10.1128/AAC.47.2.689-696.2003
[53] Wang S. Ordered mesoporous materials for drug delivery. Micropor Mesopor Mat 2009; 117(1-2): 1-9. https://doi.org/10.1016/j.micromeso.2008.07.002

[54] Yu LX, Wang JT, Hussain AS. Evaluation of USP apparatus 3 for dissolution testing of immediate-release products. AAPS PharmSci 2002; 4(1). https://doi.org/10.1208/ps040101

[55] Lokhandwala H, Deshpande A, Deshpande S. Kinetic modeling and dissolution profiles comparison: an overview. Int J Pharma Bio Sci 2013; 4: 728-37.

[56] Rengarajan GT, Enke D, Steinhart M, Beiner M. Stabilization of the amorphous state of pharmaceuticals in nanopores. J Mater Chem 2008; 18(22): 2537-39. https://doi.org/10.1039/b804266g

[57] Andersson J, Rosenholm J, Areva S, Lindén M. Influences of material characteristics on ibuprofen drug loading and release profiles from ordered micro- and mesoporous silica matrices. Chem Mater 2004; 16(21): 4160-67. https://doi.org/10.1021/cm0401490

[58] Genina N, Hadi B, Löbmann K. Hot Melt Extrusion as Solvent-Free Technique for a Continuous Manufacturing of Drug-Loaded Mesoporous Silica. J Pharm Sci 2018; 107(1): 149-55.

https://doi.org/10.1016/j.xphs.2017.05.039 